\title{
Adaptive Model Based Parameter Estimation, Based on Sparse Data and Frequency Derivatives
}

\author{
Dirk Deschrijver, Tom Dhaene, and Jan Broeckhove \\ Group Computational Modeling and Programming (CoMP) \\ University of Antwerp, Middelheimlaan 1, 2020 Antwerp, Belgium \\ \{dirk.deschrijver, tom.dhaene, jan.broeckhove\}@ua.ac.be
}

\begin{abstract}
Rational functions are often used to model and to interpolate frequency-domain data. The data samples, required to obtain an accurate model, can be computationally expensive to simulate. Using the frequency derivatives of the data significantly reduces the number of support samples, since they provide additional information during the modeling process. They are particularly useful when the data is sparse and the samples are selected adaptively.
\end{abstract}

\section{Introduction}

The simulation of complex structures can be computationally very expensive and resource-demanding. One often wants to minimize the number of costly data samples, in order to obtain an accurate model in an acceptable amount of time [1. The computational cost of higher-order derivatives is sometimes only a fraction of the cost of simulating additional samples. Some Finite Element Method (FEM) simulators, such as the High Frequency Structure Simulator (HFSS) 2] can take advantage of this property.

The Model Based Parameter Estimation (MBPE) 3] 4 is an efficient technique to model Linear Time Invariant systems (LTI) in the frequency domain using a rational pole-zero function. By expanding the numerator and denominator in a Forsythe polynomial basis [5], some numerical instabilities are improved, since this makes the normal equations best conditioned [6]. Furthermore, by using multiple spline interpolation with adaptive knot placement [7, the numerical stability issues of highly complex multi-pole systems can be circumvented.

In this paper, we extend MBPE so that the frequency derivatives of the data can be taken into account.

\section{Model Based Parameter Estimation}

\subsection{Rational Model}

Using the Model Based Parameter Estimation (MBPE) technique, the frequency domain data can be modeled by a rational transfer function :

$$
H\left(f_{s}\right)=\frac{\sum_{n=0}^{N} N_{n} \cdot\left(j 2 \pi f_{s}\right)^{n}}{\sum_{d=0}^{D} D_{d} \cdot\left(j 2 \pi f_{s}\right)^{d}}
$$


or equivalently :

$$
\left(\sum_{d=0}^{D} D_{d} \cdot\left(j 2 \pi f_{s}\right)^{d}\right) \cdot H\left(f_{s}\right)=\sum_{n=0}^{N} N_{n} \cdot\left(j 2 \pi f_{s}\right)^{n}
$$

where the $H\left(f_{s}\right)$ represent the data samples simulated at the discrete frequency $f_{s}, \forall s=0, \ldots, S . N$ and $D$ are the order of numerator and denominator respectively. Coefficient $D_{0}$ can be set to 1 without loss of generality, since it is always possible to convert to this form by dividing numerator and denominator by $D_{0}$.

The calculation of numerator and denominator coefficients in this polynomial basis (the power series) leads to an ill-conditioned Vandermonde system. For highly dynamic systems, severe numerical problems will make the results inaccurate and completely useless. Therefore, the numerator and denominator of the rational function will be decomposed in a separate basis of Forsythe polynomials. This approach guarantees that the set of normal equations are best conditioned [8].

\subsection{Forsythe Polynomials}

The Forsythe polynomials are derived from the following three-term recurrence relation :

$$
\begin{aligned}
p_{-1}(f)= & 0 \\
P_{0}(f)= & 1 \\
P_{1}(f)= & j 2 \pi f \cdot p_{0}(f) \\
& \cdots \\
P_{i}(f)= & j 2 \pi f \cdot p_{i-1}(f)+\beta_{i-1} \cdot p_{i-2}(f)
\end{aligned}
$$

The coefficients $\beta$ are calculated by summation over all negative and positive sample frequencies

$$
\beta_{i}=\sqrt{\sum_{s=0}^{S}\left[P_{i}\left(f_{s}\right)\right]\left[P_{i}\left(f_{s}\right)\right]^{*}}
$$

which leads to the following orthonormal Forsythe polynomials

$$
p_{i}(f)=\frac{P_{i}(f)}{\beta_{i}}
$$

The polynomials are orthonormal over the discrete sample set $f_{s}$, such that

$$
\sum_{s=0}^{S}\left[p_{m}\left(f_{s}\right)\right] \cdot\left[p_{n}\left(f_{s}\right)\right]^{*}=\delta_{m n} \quad \forall 0 \leq m, n<S
$$

After performing the basis transformation, the coefficients of numerator and denominator can be calculated by solving the following set of equations for all frequencies $f_{s}$ with a Singular Value Decomposition (SVD) :

$$
A_{s} x=b_{s}
$$

where 


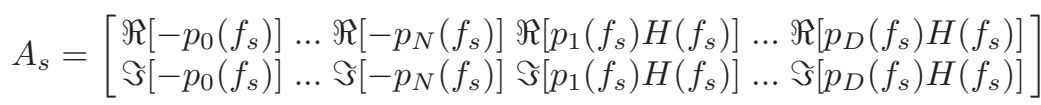

$$
\begin{aligned}
& x=\left[\begin{array}{llllll}
N_{0} & \ldots & N_{N} & D_{1} & \ldots & D_{d}
\end{array}\right]^{T} \\
& b_{s}=\left[\begin{array}{l}
\Re\left[-p_{0}\left(f_{s}\right) H\left(f_{s}\right)\right] \\
\Im\left[-p_{0}\left(f_{s}\right) H\left(f_{s}\right)\right]
\end{array}\right]
\end{aligned}
$$

Note that the system can be overdetermined, such that the number of rows in matrix A exceeds the number of columns (or the number of unknown coefficients).

\section{Derivatives}

Now assume that frequency derivatives of the data are available at the discrete frequencies $f_{s}$.

If $p_{i}^{(t)}\left(f_{s}\right)$ represents the $\mathrm{t}^{\text {th }}$ derivative of the $\mathrm{i}^{\text {th }}$ Forsythe polynomial, evaluated in frequency $f_{s}$, it can be expressed as :

$$
p_{i}^{(t)}\left(f_{s}\right)=\frac{t \cdot p_{i-1}^{(t-1)}\left(f_{s}\right)+j 2 \pi f \cdot p_{i-1}^{t}\left(f_{s}\right)+\beta_{i-1} \cdot p_{i-2}^{(t)}\left(f_{s}\right)}{\beta_{i}}
$$

To avoid a breakdown of the orthogonality, virtual samples are introduced when frequency derivatives are used.

Equation (2) can be generalized by taking the frequency derivatives into account. The coefficients $N_{n}$ and $D_{n}$ of the rational fitting model now satisfy :

$$
\begin{aligned}
H^{(t)}\left(f_{s}\right) \cdot \sum_{d=0}^{D} D_{d} \cdot p_{d}^{(0)}\left(f_{s}\right)= & \sum_{n=t}^{N} N_{n} \cdot p_{n}^{(t)}\left(f_{s}\right) \\
& -\sum_{k=1}^{t} \sum_{d=k}^{D} D_{d} \cdot p_{d}^{(k)}\left(f_{s}\right) \cdot H^{(t-k)}\left(f_{s}\right) \cdot \frac{t !}{k !(t-k) !}
\end{aligned}
$$

where $p_{n}^{(t)}$ is the $\mathrm{t}^{t h}$ order derivative of the $\mathrm{n}^{t h}$ order numerator Forsythe polynomial, $p_{d}^{(t)}$ is the $\mathrm{t}^{\text {th }}$ order derivative of the $\mathrm{d}^{t h}$ order denominator Forsythe polynomial, and $H^{(t)}$ is the $\mathrm{t}^{\text {th }}$ order derivative of the frequency domain data. All derivatives are relative to $j 2 \pi f$. The set of equations at all frequencies $f$ and for all derivatives $t$, can be written in a matrix form, similar to equation (7).

In some cases, the magnitude of the derivatives can be extremely small or large. Calculating the coefficients using all the information can make the corresponding least-squares system ill-conditioned. Therefore it is recommended to check the magnitude of the derivatives before they are used. A trade-off needs to be made between the loss of information and the loss of numerical stability. 


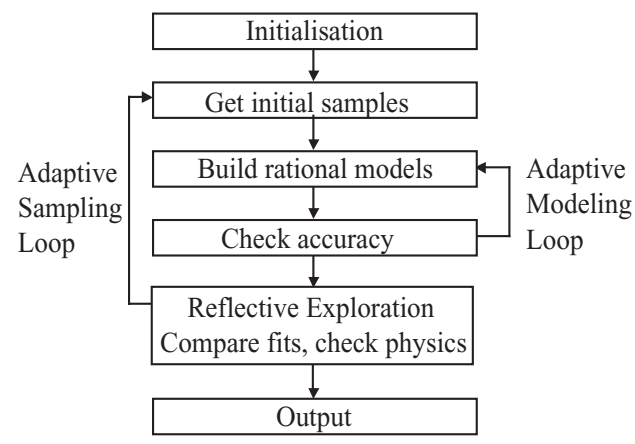

Fig. 1. Flowchart of the FFS algorithm

\section{Fast Frequency Sweep}

To minimize the cost of simulating computationally expensive data samples, an adaptive algorithm is used that automatically selects a minimal sample distribution and model complexity [1].

The flow chart of the algorithm is shown in Figure 1. It consists of an adaptive modeling loop, and an adaptive sample selection loop.

The algorithm starts with 4 samples equidistantly spaced over a certain frequency range of interest. Depending on the number of available data samples, multiple rational models are built with different order of numerator and denominator, exploiting all degrees of freedom.

The rational fitting models are evaluated in the data points, and compared against one another. If the error between the rational model (and its derivatives) evaluated in the selected sample points and the simulated data samples (and its derivatives), exceeds a certain threshold, the model is rejected, and the model's complexity is increased. All models with different order of numerator and denominator are ranked, and the 2 best models (i.e. with lowest overall error) are retained.

The difference between the two models is called the estimated fitting error, and new samples should be chosen in such way, that the maximum estimated fitting error is minimized. A reliable way to estimate the fitting error, and select new samples is given in [1]. Optionally, the algorithm can be extended with an adaptive spline selection loop, as introduced in [7].

\section{Example: Ring Resonator}

The new proposed technique is used to model the reflection coefficients $S_{11}$ of a microwave RingResonator over the frequency range $[1 \mathrm{GHz}-1.2 \mathrm{GHz}]$. The desired accuracy level is $-60 \mathrm{~dB}$, which corresponds to 3 significant digits. A highly accurate full-wave electro-magnetic simulator Momentum [9] is used to simulate the sparse data samples. 




Fig 2a.

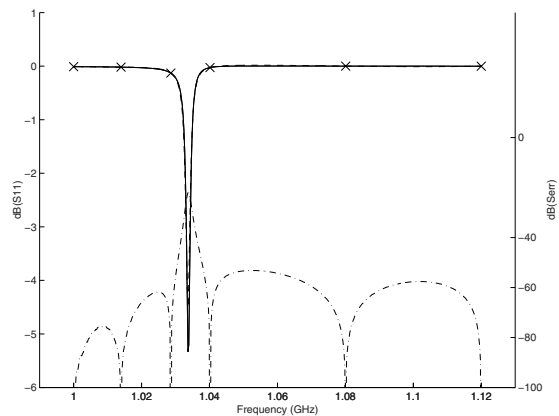

Fig 2c.



Fig $2 b$.



Fig 2d.

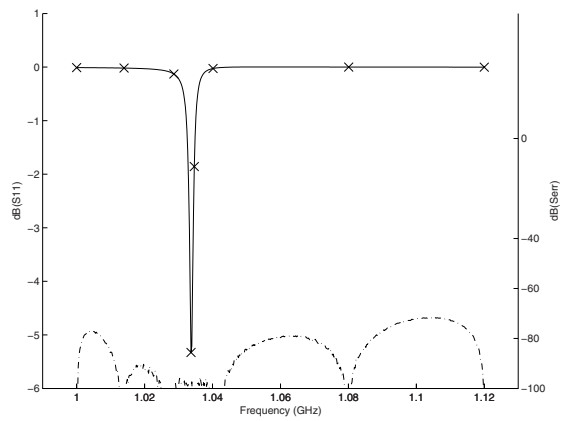

Fig 2e.

Fig. 2. Multiple steps of adaptive modeling process of a RingResonator, based on a sparse set of data samples (no frequency derivatives). The desired reference model is represented by a solid line. The selected samples are marked with a cross, and the "best" intermediate model is plotted as a dashed line. The real modeling error is plotted as a dash-dotted line (right axis).

In Figure 2a-e, the component is modeled using the default MBPE, i.e. without making use of frequency derivatives. The algorithm initially starts with 4 samples, equidistantly spaced over the frequency range of interest, and builds several interpolation models. The "best" model is shown as a dashed line. In 




Fig 3a.



Fig 3b.

Fig. 3. Multiple steps of adaptive modeling process of a RingResonator, based on a sparse set of data samples + first frequency derivatives. The desired reference model is represented by a solid line. The selected samples are marked with a cross, and the "best" intermediate model is plotted as a dashed line. The real modeling error is plotted as a dash-dotted line (right axis).



Fig. 4. Multiple steps of adaptive modeling process of a RingResonator, based on a sparse set of data samples + first and second frequency derivatives. The desired reference model is represented by a solid line. The selected samples are marked with a cross, and the "best" intermediate model is plotted as a dashed line. The real modeling error is plotted as a dash-dotted line (right axis).

each iteration of the algorithm, new samples are selected where the estimated error (the difference between 2 rational approximants, based on the same set of support samples) is maximal. Based on the extra data points, new rational models are built and evaluated, and the estimated error function is updated. The iterative process is repeated until the error is below a predefined accuracy threshold. In this example, 8 support samples are automatically selected, to obtain the required precision.

In Figure 3a-b, the first frequency derivatives are also used in the modeling process. This additional information is exploited by the algorithm, and now it 
only needs 5 samples to find an accurate model. In this example, the extra sample was chosen very efficiently in the dip of the resonance.

In Figure 4, the second frequency derivatives are also used in the modeling process. The initial set of equally distributed samples already guarantee sufficient accuracy.

Of course, it can be useful to take also higher-order derivatives into account, especially if the behaviour of the system is highly dynamic. Typically, if more derivatives are used, fewer samples will be needed to get an accurate model. However, the value of the higher order derivatives in determining the model parameters is usually less than the value of lower order derivatives or samples, partially due to numerical issues.

\section{Conclusion}

In this paper, we extend the MBPE technique with frequency derivatives. During the adaptive modeling process, this reduces the number of required support samples significantly. This approach is particularly useful if the computational cost of simulating the required support samples is very high, and when the samples are selected adaptively. And so, an accurate model can be obtained, using a minimal set of data samples and their frequency derivatives. The stability of the algorithm is improved by a transformation of the rational model into a generalized Forsythe orthonormal basis, which makes the normal equations of the least squares system best conditioned.

Acknowledgement. This work was supported by the Fund for Scientific Research-Flanders.

\section{References}

1. Dhaene, T.: Automated Fitting and Rational Modeling Algorithm for EM-based Sparameter data. LNCS 2367, Springer-Verlag, PARA 2002, Espoo (Finland) (2002) 99-105

2. Ansoft: Ansoft HFSS, Ansoft Corporation, Pittsburgh, PA

3. Miller, E. K.: Model Based Parameter Estimation in Electromagnetics: Part I. Background and Theoretical Development. IEEE Antennas and Propagation Magazine 40(1) (1998) 42-51

4. Miller, E. K.: Model Based Parameter Estimation in Electromagnetics: Part II. Application to EM observables. Applied Computational Electromagnetics Society Newsletter, 11(1) (1996) 35-56

5. Forsythe, G. E.: Generation and use of Orthogonal Polynomials for Data-Fitting with a Digital Computer. Journal of SIAM 5(2) (1957) 74-88

6. Vandersteen, G.: Curve Fitting using Splines, Polynomials and Rational approximations : a comparative study. NORSIG 96, IEEE Nordic Signal Processing Symposium, Espoo (Finland) (1996) 41-44 
7. Deschrijver, D., Dhaene, T.: Adaptive Knot Placement for Rational Spline Interpolation of Sparse EM-Based data. ICECOM 03, 17th International Conference on Applied Electromagnetics and Communications, Dubrovnik (Croatia) (2003) 433436

8. Forsythe, G. E., Straus, E.G.: On Best Conditioned Matrices. Proceedings of the American Mathematical Society, 6 (1955) 340-345

9. Agilent EEsof Comms EDA: Momentum Software, Agilent Technologies, Santa Rosa, CA 